Editor's Note: These short, critical reviews of recent papers in the Journal, written exclusively by graduate students or postdoctoral fellows, are intended to summarize the important findings of the paper and provide additional insight and commentary. For more information on the format and purpose of the Journal Club, please see http://www.jneurosci.org/misc/ifa_features.shtml.

\title{
Reactivation in Ventral Striatum during Hippocampal Ripples: Evidence for the Binding of Reward and Spatial Memories?
}

\author{
Omar Jamil Ahmed, ${ }^{1}$ James McFarland, ${ }^{2}$ and Arvind Kumar, ${ }^{1,3}$ \\ Departments of ${ }^{1}$ Neuroscience and ${ }^{2}$ Physics, Brown University, Providence, Rhode Island 02912, and ${ }^{3}$ Bernstein Center for Computational Neuroscience, \\ University of Freiburg, 79104 Freiburg, Germany \\ Review of Lansink et al. (http://www.jneurosci.org/cgi/content/full/28/25/6372)
}

Over 50 years ago, Scoville and Milner (1957) reported the case of H. M., an epileptic patient who underwent bilateral removal of his medial temporal lobe, a region of the brain that encompasses the hippocampus. After the surgery, H. M. was unable to form new memories, highlighting the importance of the hippocampus in memory formation. However, H. M. retained most of his childhood memories, suggesting that these older memories were stored outside of the hippocampus. Research on primates and rodents has corroborated the essential role of the hippocampus in transforming new memories into stable representations elsewhere in the brain (Squire, 1992), a process known as consolidation. However, the mechanisms underlying consolidation remain poorly understood, and it is these fundamental mechanisms that a recent study by Lansink et al. (2008) in The Journal of Neuroscience attempts to understand.

Many theories of memory consolida-

Received Aug. 10, 2008; revised Aug. 24, 2008; accepted Aug. 25, 2008.

This work was supported by National Institute of Mental Health Predoctoral Grant MH081477 (0.J.A.). We thank Dr. Scott Cruikshank, Anne Booker, Vivek Buch, and Samuel Reiter for a careful reading of this manuscript.

Correspondence should be addressed to Omar Jamil Ahmed, Department of Neuroscience, Brown University, 185 Meeting Street, Box G-LN, Providence, RI 02912.E-mail: omar_ahmed@brown.edu.

DOI:10.1523/JNEUROSCI.3778-08.2008

Copyright $\odot 2008$ Society for Neuroscience $\quad$ 0270-6474/08/289895-03\$15.00/0 tion propose the reactivation of behavioral experiences, typically during periods of sleep or rest. Electrophysiological evidence for such theories has been recorded in hippocampal place cells in rats. Pavlides and Winson (1989) found that after a rat was exposed to a particular region of space, only place cells encoding that specific region increased their firing rates during postbehavioral sleep (POST). Wilson and McNaughton (1994) extended this finding to show that pairs of place cells that had correlated activity during a track session (RUN) were more strongly correlated during POST sleep, a phenomenon called reactivation of correlated activity. More complex examples of behavioral activity recurring during POST sleep have also been observed. For instance, specific multineuron activity patterns during RUN are reactivated during both rapid eye movement (REM) sleep (Louie and Wilson, 2001) and slow-wave sleep (Lee and Wilson, 2002), a phenomenon known as replay. Replay thus refers to a particular sequence of spikes among a population of cells, whereas reactivation refers to the simultaneous activity of pairs of cells.

Active and sleep states are often defined by their cortical and hippocampal EEG or local field potential (LFP) signals. As a rat runs on a track, LFP recordings in the hippocampus reveal an $8 \mathrm{~Hz}$ extracellular oscillation, called the theta oscilla- tion. REM sleep is also characterized by 8 $\mathrm{Hz}$ theta oscillations in the hippocampus. Given this similarity, it is perhaps unsurprising that replay of RUN activity in REM sleep occurs on almost the same time scale as the original spike patterns seen during RUN (Louie and Wilson, 2001).

During slow-wave sleep, the cortex shows prominent slow $(0.5-3 \mathrm{~Hz})$ oscillations, but the hippocampal LFP is characterized by high-amplitude irregular activity. These irregular hippocampal events are marked by large, brief (100 ms), highfrequency oscillations called ripples. Ripples are accompanied by the synchronous firing of many hippocampal cells, and are hence thought to be important in consolidation. Amazingly, hippocampal activity patterns that span several seconds during RUN are compressed 20-fold when they are replayed during a single ripple event in slow-wave sleep (Lee and Wilson, 2002). The reactivation of correlated activity first observed by Wilson and McNaughton (1994) also occurs preferentially during ripple events. Thus, behaviorally induced activity can recur in different forms during sleep, and ripples are important in most cases.

Many theories of memory consolidation suggest that multiple brain regions are simultaneously reactivated so that multimodal information can be "bound" together into unified memories. Other 
brain regions would thus be expected to show concurrent reactivation with the hippocampus to strengthen memories stored outside the hippocampus. Indeed, replay has been recorded simultaneously in the hippocampus and the visual cortex (Ji and Wilson, 2007). Furthermore, Pennartz et al. (2004) showed that neurons in the ventral striatum are reactivated during quiet wakefulness/slow-wave sleep (QWSWS). This striatal reactivation is coincident with hippocampal ripples, suggesting that the ventral striatum is also involved in more global consolidation processes.

The ventral striatum encompasses the nucleus accumbens, a region that is thought to encode both reward expectation and reward delivery, and might also play a role in controlling motor output. Given this reward-related activity, Lansink et al. (2008) asked whether ventral striatum reactivation is specific to rewardmodulated cells, and could thus contribute a motivational component to the memories being consolidated.

Like Pennartz et al. (2004), Lansink et al. (2008) addressed these questions by using a measure of reactivation known as explained variance (EV). To calculate EV, the authors record the activity of a population of ventral striatal neurons in three epochs: prebehavioral sleep (PRE), RUN, and POST. By calculating all possible pairwise correlations among the recorded cells, EV measures how well the correlations during RUN predict the correlations during POST. As a control, the "reverse explained variance" (REV) is calculated by replacing POST with PRE. EV thus provides a population-based measure of the pairwise reactivation found by Wilson and McNaughton (1994).

Cells were classified as reward cells or nonreward cells based on whether they showed a significant response time locked to a reward site visit [Lansink et al. (2008), their Fig. 2 (http://www.jneurosci.org/cgi/ content/full/28/25/6372/F2)]. As found by Pennartz et al. (2004), cells in the ventral striatum showed significant reactivation (defined as EV > REV), and this reactivation persisted for at least $40 \mathrm{~min}$ during POST [longer than what has been observed in the hippocampus (Wilson and McNaughton, 1994)]. Interestingly, ventral striatum reactivation was limited to QWSWS, and did not occur during REM sleep [Lansink et al. (2008), their Fig. 4 (http:// www.jneurosci.org/cgi/content/full/28/25/ 6372/F4)]. Furthermore, significant reactivation during QW-SWS was confined to $200 \mathrm{~ms}$ periods after the onset of hippocam-

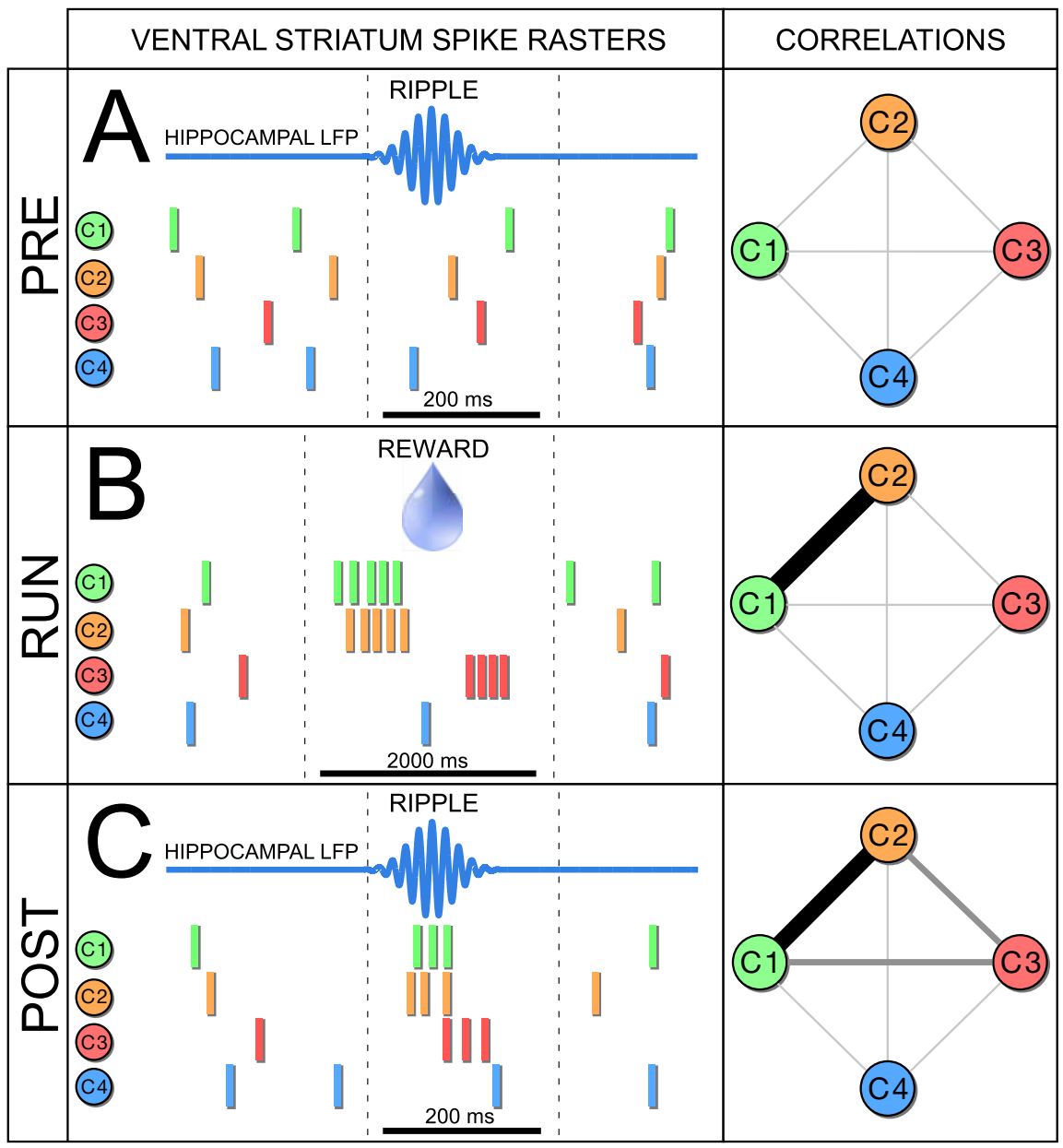

Figure 1. Schematic depiction of the main findings of Lansink et al. (2008). The hypothetical activities of four ventral striatal cells (C1-C4) during PRE, RUN, and POST are shown in the left column. The corresponding pairwise correlations are depicted by the thickness of the lines connecting the cells and are shown in the right column. $A$, During PRE, all four cells fire at a constant rate, and their firing rate does not change during a ripple in the hippocampal local field potential (blue trace). $\boldsymbol{B}$, During RUN, the reward (indicated by a water drop) elicits strong responses in cells $\mathrm{C} 1$ (green), C2 (orange), and C3 (red), but not in cell C4 (blue). Of the three reward cells, only $\mathrm{C} 1$ and $\mathrm{C} 2$ show the same temporal response to the reward and are thus strongly correlated during the RUN period. C, In POST, all three reward cells (C1-C3) fire preferentially during the hippocampal ripple. However, the two cells that were strongly correlated on the track are the most strongly correlated during POST ripples. Because of the strong correlation of C1 and $\mathrm{C} 2$ during both RUN and POST, the EV is high, and the C1/C2 cell pair is said to be strongly reactivated.

pal ripples [Lansink et al. (2008), their Fig. 3 (http://www.jneurosci.org/cgi/content/full/ 28/25/6372/F3)].

To quantify the specific contribution of reward cells to reactivation, the authors pooled their data across rats and across sessions, because the number of reward cells per session was typically limited. To do this, pairs of reward cells were sampled from the pooled data, each sample consisting of the pair's activity over PRE, RUN, and POST. Both nonreward- and reward-cell populations showed significant reactivation. However, reward-cell populations showed greater differences between EV and REV [Lansink et al. (2008), their Fig. 5A (http://www.jneurosci. org/cgi/content/full/28/25/6372/F5)], indicating that reward cells are more strongly reactivated. Reward cells also demonstrated an increased tendency to fire spikes during hippocampal ripple events in POST QW-SWS relative to PRE QW-SWS, which could not be explained by overall changes in firing rate (Fig. 1).

The realignment of ventral striatal reward-cell spikes with POST hippocampal ripples was quite substantial. In PRE, reward cells fired at the same rate, both during ripples and between ripples (Fig. $1 A$, cells $1-3)$. In POST, however, the firing rate during ripples was double the rate between ripples (Fig. 1C, cells 1-3). This realignment of spikes to coincide with ripples was distinctly absent in nonreward cells (Fig. 1A,C, cell 4). Such a realignment of reward-cell spikes with brief ripple events should increase the correlations between pairs of reward cells in POST compared with PRE. As the authors point 
out, the large reactivation in reward-cell populations was shown to be entirely dependent on a small subset (5 of 70) of strongly correlated reward-cell pairs [Lansink et al. (2008), their Fig. 5B (http:// www.jneurosci.org/cgi/content/full/28/ 25/6372/F5)]. This suggests that although most reward cells realigned with POST ripples, the reward cells that were strongly correlated during RUN were the most strongly correlated during the brief POST ripples. Thus, correlations between reward cells during RUN seem to be preserved within the compressed time frame of POST ripples (Fig. $1 B, C$, cells 1,2 ).

Is this reactivation attributable to strengthened local connections within the ventral striatum or changes in extrinsic inputs? All principal cells in the ventral striatum are inhibitory. These cells primarily project to regions outside the ventral striatum, but also branch extensively within the ventral striatum, providing recurrent inhibitory input to other principal cells. This lateral inhibition is weaker than the feedforward inhibition from fastspiking GABAergic interneurons (Tepper et al., 2004). Given this predominance of inhibitory connectivity in the ventral striatum, it might seem unlikely that the coordinated reactivation seen in POST is caused by the strengthening or weakening of local connections during RUN. However, many studies have shown that mutually inhibited neurons can show strongly correlated firing (White et al., 1998). Furthermore, $2 \%$ of cells in the ventral striatum are giant cholinergic interneurons that densely innervate and strongly modulate the firing of principal cells. These cholinergic cells are themselves modulated by motivationally relevant dopaminergic projections from the ventral tegmental area. Lansink et al. (2008) excluded fast-spiking interneurons from their analysis, but did not distinguish between principal and cholinergic cells. However, it might be possible to identify cholinergic cells based on their tonic baseline firing, broad spikes, and large afterhyperpolarization (Zhou et al., 2002) and to assess whether their firing rates change during POST ripples. It is possible that altered cholinergic activity facilitates the reactivation of reward-related principal cells. Alternatively, the preferential re- ward cell firing during POST ripples could be primarily driven by extrinsic inputs from the hippocampus, prefrontal cortex, or amygdala, all of which project directly to the ventral striatum.

We wish to reemphasize one of the most important findings of the Lansink et al. (2008) study: ventral striatal cells that showed reward modulation in the RUN session preferentially fired during hippocampal ripples in POST, but not in PRE. This is a striking result, but how could it come about? It may be informative to consider the firing patterns of both ventral striatal and hippocampal cells during a RUN session on the track. As a rat runs, hippocampal place cells fire in a particular sequence. However, as the rat approaches the reward and slows down, the hippocampal LFP transitions from theta oscillations to ripples (Diba and Buzsáki, 2007). Compressed replay, or reverse replay, of hippocampal place cells can even occur during these rewardassociated ripples on the track (Diba and Buzsáki, 2007). Thus, even during RUN, it is possible that ventral striatal reward-cell activity is accompanied by, and perhaps driven by, hippocampal ripple-associated replay. This coordinated firing between hippocampal place cells and ventral striatal reward cells during RUN ripples could strengthen the connections between the ventral striatal representation of the reward and the place cells encoding the trajectory that led to the reward. During POST sleep, a replay of this hippocampal activity during ripples would then be expected to selectively drive reactivation of reward cells in the ventral striatum via the strengthened hippocampal projections to these cells.

Testing the relationship between hippocampal place cell firing, hippocampal ripples, and reward-cell activity in the ventral striatum during RUN sessions thus represents an important next step. It is possible that the binding of hippocampal and ventral striatal memories has already begun on the track, and the coordinated firing of reward cells in ventral striatum during POST hippocampal ripples consolidates this information during subsequent slow-wave sleep. The ventral striatum is thought to bind spatial information from the hippocampus, emo- tional information from the amygdala, and higher-order information from the prefrontal cortex and to send this processed information to the rest of the basal ganglia, which can then modulate the motor output of the animal. Thus, the results of Lansink et al. (2008) have profound implications for how binding of multimodal information can take place, and are an important advance in our understanding of the electrophysiological signatures of consolidation.

\section{References}

Diba K, Buzsáki G (2007) Forward and reverse hippocampal place-cell sequences during ripples. Nat Neurosci 10:1241-1242.

Ji D, Wilson MA (2007) Coordinated memory replay in the visual cortex and hippocampus during sleep. Nat Neurosci 10:100-107.

Lansink CS, Goltstein PM, Lankelma JV, Joosten RN, McNaughton BL, Pennartz CM (2008) Preferential reactivation of motivationally relevant information in the ventral striatum. J Neurosci 28:6372-6382.

Lee AK, Wilson MA (2002) Memory of sequential experience in the hippocampus during slow wave sleep. Neuron 36:1183-1194.

Louie K, Wilson MA (2001) Temporally structured replay of awake hippocampal ensemble activity during rapid eye movement sleep. Neuron 29:145-156.

Pavlides C, Winson J (1989) Influences of hippocampal place cell firing in the awake state on the activity of these cells during subsequent sleep episodes. J Neurosci 9:2907-2918.

Pennartz CM, Lee E, Verheul J, Lipa P, Barnes CA, McNaughton BL (2004) The ventral striatum in off-line processing: ensemble reactivation during sleep and modulation by hippocampal ripples. J Neurosci 24:6446-6456.

Scoville WB, Milner B (1957) Loss of recent memory after bilateral hippocampal lesions. J Neurol Neurosurg Psychiatry 20:11-21.

Squire LR (1992) Memory and the hippocampus: a synthesis from findings with rats, monkeys, and humans. Psychol Rev 99:195-231.

Tepper JM, Koós T, Wilson CJ (2004) GABAergic microcircuits in the neostriatum. Trends Neurosci 27:662-669.

White JA, Chow CC, Ritt J, Soto-Treviño C, Kopell N (1998) Synchronization and oscillatory dynamics in heterogeneous, mutually inhibited neurons. J Comput Neurosci 5:5-16.

Wilson MA, McNaughton BL (1994) Reactivation of hippocampal ensemble memories during sleep. Science 265:676-679.

Zhou FM, Wilson CJ, Dani JA (2002) Cholinergic interneuron characteristics and nicotinic properties in the striatum. J Neurobiol 53:590-605. 